\title{
Reduction of Parameter Sensitivity in an Induction Motor Current Regulator Using Integrated Pilot Sensors in the Low-Side Switches
}

\author{
Sibaprasad Chakrabarti, Member, IEEE, Thomas M. Jahns, Fellow, IEEE, and Robert D. Lorenz, Fellow, IEEE
}

\begin{abstract}
A closed-loop synchronous-frame current regulator for an induction machine drive that is capable of delivering high-quality performance characteristics using integrated pilot current sensors in the three low-side switches of a three-phase bridge inverter has been developed. However, the necessary current reconstruction exhibits sensitivity to errors in the machine parameters, which has been documented during this investigation. This paper presents a technique for introducing a gated integrator into the controller gain amplifier that compensates for parameter errors using feedback information from all three of the phase currents during the periodic intervals when it is available. A numerical singularity in the current reconstruction algorithm during light-load operation is overcome by introducing an alternative first-order current estimator that is activated over narrow angular spans of the fundamental component vector rotation. This paper also presents an in situ technique to compensate gain differences between the three pilot devices in the inverter unit. The effectiveness of these improvements is verified using experimental results.
\end{abstract}

Index Terms-Decoupling voltage, indirect field-oriented control, integrated pilot current sensor, three-phase induction motor drives.

\section{INTRODUCTION}

$\mathbf{S}$ IGNIFICANT efforts are under way internationally to find improved techniques to reduce the cost and size of inverters by integrating more functionality inside the power modules. This trend is creating renewed interest in the use of integrated pilot current sensors that are directly incorporated into MOSgated power semiconductor switches to measure the phase currents for feedback purposes.

Past research [1]-[3] has shown that pilot current sensors can be successfully applied to achieve closed-loop current regulation in some types of brushless machines with trapezoidal cur-

Paper IPCSD-05-042, presented at the 2004 Industry Applications Society Annual Meeting, Seattle, WA, October 3-7, and approved for publication in the IEEE TRANSACTIONS ON INDUSTRY APPLiCATIONS by the Industrial Drives Committee of the IEEE Industry Applications Society. Manuscript submitted for review May 1, 2004 and released for publication July 14, 2005. This work was supported by the ERC Program of the National Science Foundation under Award EEC-9731677 and by the Center for Power Electronic Systems (CPES) and the Wisconsin Electric Machines and Power Electronics Consortium (WEMPEC), both of the University of Wisconsin, Madison.

S. Chakrabarti is with GE Transportation Systems, Erie, PA 16531 USA (e-mail: sibaprasad.chakrabarti@ge.com).

T. M. Jahns is with the Department of Electrical and Computer Engineering, University of Wisconsin, Madison, WI 53706 USA (e-mail: jahns@engr.wisc. edu; t.jahns@ieee.org).

R. D. Lorenz is with the Department of Mechanical Engineering and the Department of Electrical and Computer Engineering, University of Wisconsin, Madison, WI 53706 USA (e-mail: lorenz@engr.wisc.edu).

Digital Object Identifier 10.1109/TIA.2005.857475

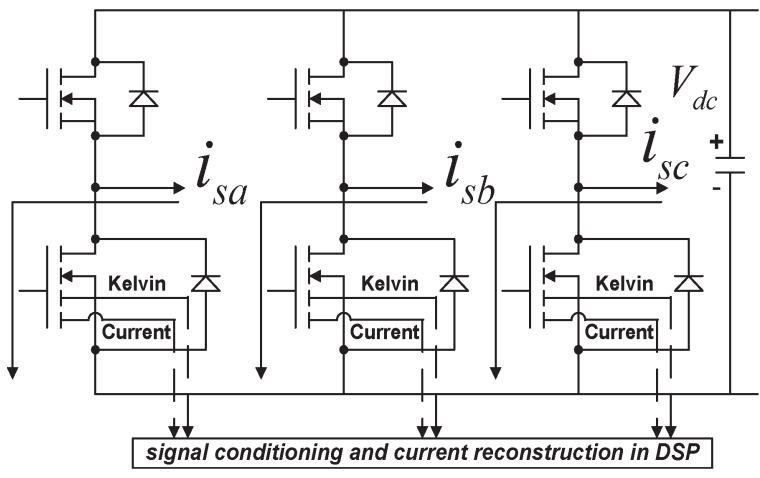

Fig. 1. Three-phase voltage-source pulsewidth-modulation (PWM) inverter with integrated pilot current sensors in the three low-side switches.

rent excitation including permanent magnet "brushless direct current (dc)" and switched reluctance machines. In addition, other research [4] has demonstrated that $n$-channel insulatedgate bipolar transistor (IGBT) devices can be designed with pilot current sensors that have sufficient accuracy and linearity to enable their use as the sensing elements in closed-loop current regulators.

Three-phase sinusoidal excitation creates complications for using an inverter with pilot current sensors installed only in the three low-side switches (see Fig. 1) since there are periodic intervals when only one of the three phase currents is measurable. This makes it necessary to reconstruct (or estimate) the missing phase currents.

Recent work [5] has shown that it is possible to achieve highquality closed-loop current regulator performance with threephase passive $R L$ loads using pilot sensors in the low-side switches. Unfortunately, this approach does not work well with three-phase induction machine loads because of the need to independently control the magnetizing and the torque-producing components of the stator current (i.e., $i_{s d}$ and $i_{s q}$ ).

More recently, a technique has been developed to achieve closed-loop current regulation in an induction machine that yields very promising results for operation with pilot current sensors in the low-side switches [6]. More specifically, this new current control technique has been successfully applied to implement indirect field-oriented control (IFOC) for the induction machine using rotor speed information from an encoder.

As documented in an earlier paper [6], this new algorithm is vulnerable to a numerical singularity that appears at light load, determined by the ratio of the flux- and torqueproducing components of the stator currents. More specifically, 
numerical computation of the reciprocal sine function at light load becomes extremely difficult for a narrow angular span around the singularity point, especially when the control algorithm is implemented using a fixed-point digital signal processor (DSP).

Separately, the variability of the current sensing gain among the pilot sensors in a group of switch device units can degrade the current regulator performance. Unless compensated, different gains in the three pilot sensors in Fig. 1 will produce undesirable ripple torque in the machine because of the current measurement inaccuracies.

One of the major objectives of this paper is to directly investigate the sensitivity of the new current regulator performance to errors in knowledge of the induction machine parameters and techniques for reducing this sensitivity (Section III). The structure of the synchronous-frame current regulator algorithm using the pilot current sensors is briefly reviewed. The parameter sensitivity is examined individually for each of the major induction machine model parameters.

It is shown that proper application of a gated integrator in the outer loop of the current regulator can have significant beneficial effects (Section IV). More specifically, the integrator minimizes the key parameter sensitivities in order to enhance both the steady-state and dynamic characteristics of the current regulator performance.

This paper also proposes a suitable current control scheme that supplements the original synchronous-frame current regulator for only a small angular span around the singularity point associated with light-load operation (Section V). This feature eliminates the problems associated with evaluating the reciprocal sine function in the original synchronous-frame regulator structure.

As a third contribution, this paper also proposes an accurate tuning technique to compensate for differences in the current gains of the three pilot sensors in the three-phase inverter unit (Section VI).

A review of the basic current control technique for an induction machine using pilot current sensors [6] in the three lowside inverter switches is provided in Section II.

\section{SYNCHRONOUS-FrAME CURRENT CONTROL AlgORITHM}

For the development of the synchronous-frame current controller, a voltage model of the three-phase induction motor has been developed. A key feature is to orient the rotor flux axis along the $d$-axis of the synchronously rotating frame, similar to the IFOC scheme conventionally used for the three-phase induction motor model [7], [8]. Assuming that the slip calculation is accurate at all times, the synchronous-frame stator voltage $v_{s d q}$ can be expressed as a complex vector

$$
v_{s d q}=\left(r_{s}+j \omega_{e} \sigma L_{s}\right) i_{s d q}+j \omega_{e} \frac{L_{m}^{2}}{L_{r}} i_{s d}+\sigma L_{s} \frac{\mathrm{d} i_{s d q}}{\mathrm{~d} t} .
$$

In (1), the first two terms represent the stator impedance drop and the third term represents the back electromotive force (EMF) voltage associated with the rotational speed. The fourth term accounts for the voltage drop associated with any stator

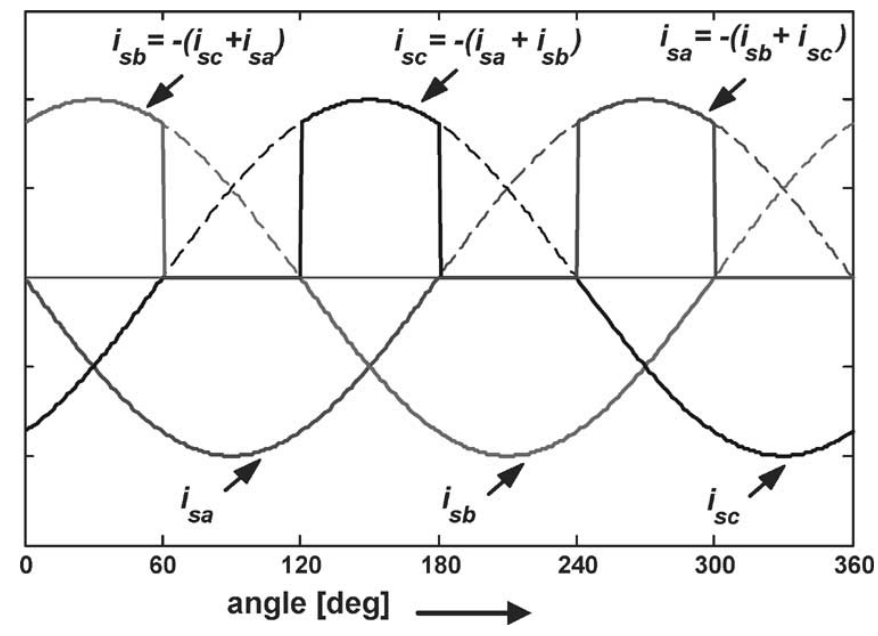

Fig. 2. Measurable phase currents using pilot sensors in low-side switches during one period. Subscript " $p$ " indicates pilot sensor current measurements and subscript " $m$ " indicates measured phase currents used as feedback in the current regulator.

current transient. The stator impedance drop and the back EMF voltages together constitute the steady-state component of the machine terminal voltage, and both terms are functions of the instantaneous current. It is well known that this steadystate machine voltage drop can be decoupled in the current regulator using the instantaneous stator current information and estimated machine parameters, which yields zero steady-state error with just a P-type controller.

The measurable phase currents with the proposed inverter topology (with only three pilot sensors in the lower side switches) are represented as a function of time in Fig. 2 for a complete fundamental cycle. This figure shows that there are three 60 electrical degree intervals when all three currents are measurable. Only one phase current is measurable during the three remaining 60 electrical degree intervals. A reconstruction algorithm has been developed [6] to reconstruct the two unmeasurable phase currents from the single measured phase current during the three intervals when only one phase current is measurable. These reconstructed phase currents are then transformed into the synchronous frame and used to calculate the decoupling voltage

$$
v_{s d q \_d}^{*}=\left(\hat{r_{s}}+j \omega_{e}^{*} \hat{\sigma} \hat{L_{s}}\right) i_{s d q \_r}+j \omega_{e}^{*} \frac{\hat{L}_{m}{ }^{2}}{{\hat{L_{r}}}} i_{s d \_r}
$$

where the subscript " $r$ " signifies reconstructed current values.

This decoupling voltage is derived inside the controller with the expectation that it will command the correct machine terminal voltage [as described in (1)] to cause the desired steady-state operating point current to flow. Of course, exact decoupling depends on accurate knowledge of the machine parameters. At this stage of the controller structure development, it is assumed that the estimated machine parameters are sufficiently accurate that this steady-state voltage decoupling between the controller and the physical machine can be achieved.

Assuming that the desired decoupling of the steady-state voltage is achieved, the transient voltage associated with any change in the stator current [represented by the last term in (1)] 


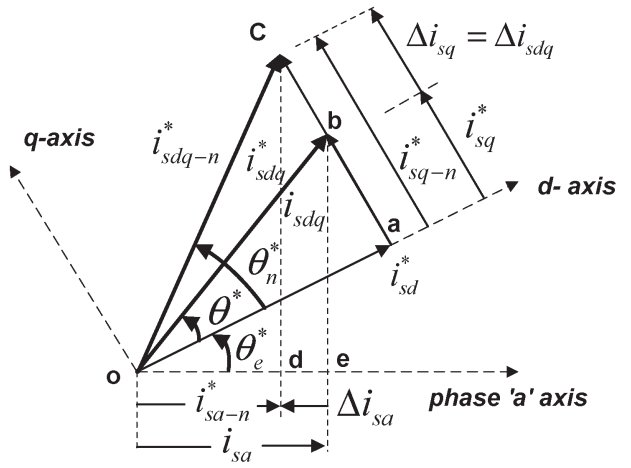

Fig. 3. Vector diagram showing complex current vectors and phase " $a$ " current component during $i_{s q}$ step transient.

can be developed by employing a suitable proportional controller that acts on the synchronous-frame current errors. The gain of this proportional controller directly determines the bandwidth of the current regulator. Nearly zero steady-state error is achieved via the accuracy of the decoupling voltage.

However, the availability of only a single measurable phase current during three 60 electrical degree intervals is insufficient to construct a synchronous-frame current regulator, even with the presence of a steady-state voltage decoupling term. A new approach has been developed [6] to estimate the $q$-axis current from the only measurable phase current assuming that the $d$-axis component of stator current command is held constant value during this interval.

Using this approach, the error between the commanded $q$-axis current and the equivalent $q$-axis machine current can be estimated even during a transient process. Fig. 3 shows that a step change in the commanded $q$-axis current creates an instantaneous angle between the commanded stator current vector $i_{s d q-n}^{*}$ (shown by vector $o c$ ) and the actual machine current $i_{s d q}$ (vector $o b$ ). Prior to this step change of the $q$-axis current command, the commanded current $i_{s d q}^{*}$ and the actual current vector $i_{s d q}$ exactly match, because it is assumed that the steady-state decoupling voltage in (2) applies exactly the correct stator voltage to cause the desired current to flow.

Assuming that the phase $a$ current is the only one that is measurable during this interval, the $q$-axis current error can be estimated using the commanded $d$-axis current vector angle $\theta_{e}^{*}$ and the measured phase $a$ current (see Fig. 3)

$$
\Delta \hat{i}_{s q}=\frac{\Delta i_{s a}}{-\sin \left(\theta_{e}^{*}\right)}=\frac{i_{s a-n}^{*}-i_{s a}}{-\sin \left(\theta_{e}^{*}\right)} .
$$

This estimation process is carried out during the interval $150^{\circ}<\left(\theta_{e}^{*}+\theta^{*}\right)<210^{\circ}$ when only the phase $a$ current is measurable. The same approach can be appropriately modified and applied during the two intervals when only phase $b$ and phase $c$ currents are measurable, respectively.

Assuming that the $d$-axis current command is unchanged, the error between the stator current vector and the actual current vector $\Delta i_{s d q}$ during the intervals when a single measurable current is available can be represented as

$$
\Delta i_{s d q}=i_{s d q}^{*}-i_{s d q}=0+j \Delta \hat{i}_{s q}
$$

Next, a synchronous-frame proportional regulator is introduced and tuned for the desired current regulator bandwidth to drive this current error towards zero. The output of this controller commands the incremental stator voltage to force the machine phase currents to change when a change in the commanded $q$-axis current occurs. The complete stator voltage vector command $v_{s d q}^{*}$, the sum of the proportional controller voltage, and the decoupling voltage can be expressed as

$$
v_{s d q}^{*}=K_{P} \Delta i_{s d q}+v_{s d q \_d}^{*}=K_{P}\left(j \Delta \hat{i}_{s q}\right)+v_{s d q \_d}^{*} .
$$

During the three $60^{\circ}$ intervals when all three phase currents can be directly derived from pilot sensor measurements, the $q$-axis error current $\Delta i_{s q}$ can be directly calculated from the measured currents and substituted for the estimated $q$-axis current error $\Delta \hat{i}_{s q}$ in (4) and (5).

Regardless of the source of the $q$-axis stator current error (i.e., measured or estimated), the synchronous-frame voltage command in (5) is finally converted to the stationary frame to provide the input commands for the PWM voltage modulator.

The proposed control algorithm has been implemented in a laboratory test demonstration using a 3-hp 240-V three-phase induction motor. The control algorithm is implemented with a fixed-point DSP that commands a PWM switching frequency of $10 \mathrm{kHz}$.

The block diagram of the implemented control algorithm is shown in Fig. 4. There are some specific blocks that have different functions compared to the corresponding blocks used in more conventional synchronous-frame current regulators. In particular, the modified blocks in Fig. 4 are as follows: 1) "reconstruction algorithm"-reconstructs the unmeasurable phase currents; 2) "dynamic voltage calculator"-determines the control voltage during the intervals when only one measured phase current is available; and 3) "toggle switch"-toggles between two proportional controller voltages depending on whether the current vector falls in an interval with either one or three measurable phase currents.

In addition, the controller also includes a block for the "decoupling voltage term," which calculates the decoupling voltage using the reconstructed currents.

\section{Sensitivity to PARAmeter Estimates}

So far, it has been assumed that the estimated machine parameters are accurate so that the decoupling voltages exactly match the required steady-state voltage of the machine. The steady-state and dynamic performance of the proposed control structure is degraded by inaccurate machine parameter estimates in the stator resistance and stator transient inductance parameters in (2). However, errors in the speed-dependent back EMF waveform do not have a significant impact on the current regulator performance [6].

Fig. 5 provides a phasor diagram that shows several voltage and current vectors developed by the current regulator for steady-state conditions during the interval when only the phase- $a$ current is measurable. Voltage vector $o g$ represents the decoupling voltage vector with accurate parameter estimates. 


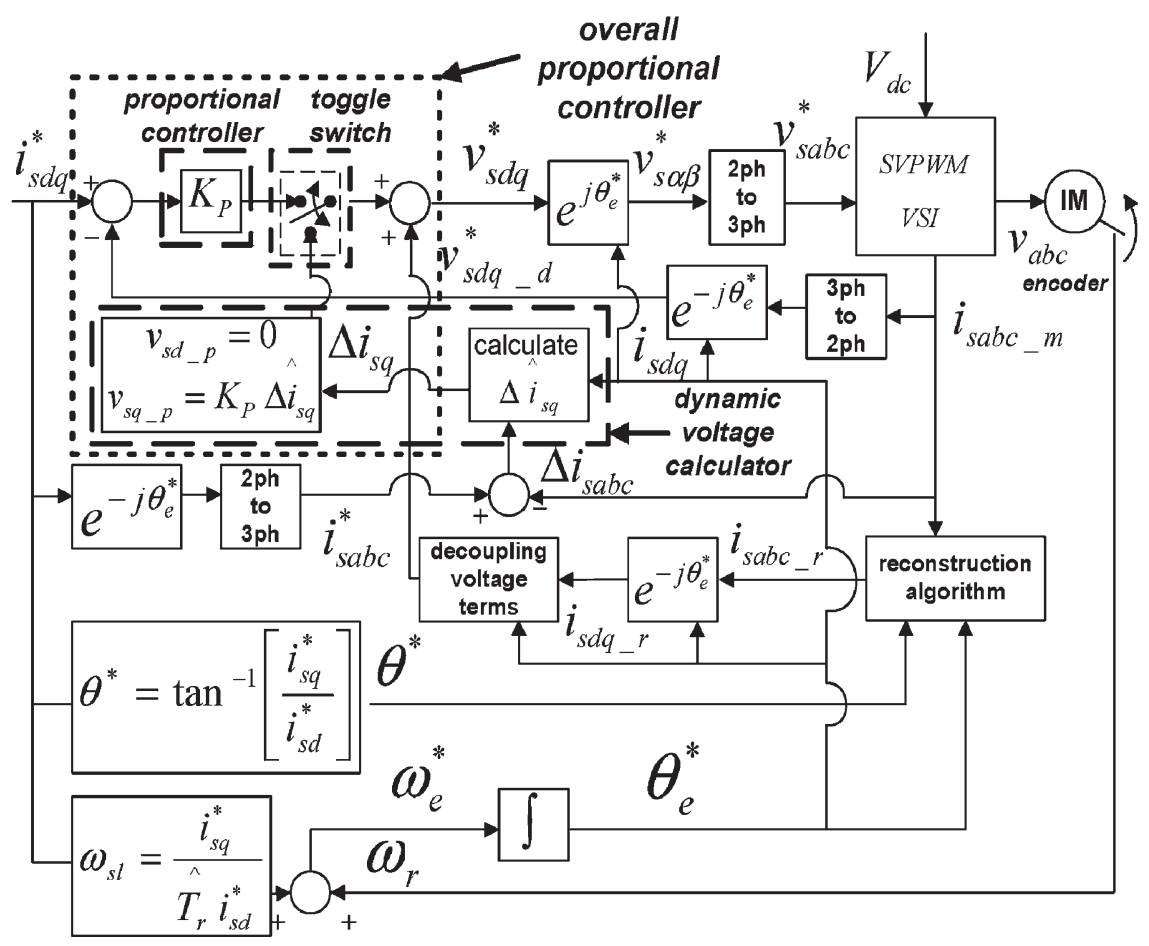

Fig. 4. Overall control block diagram for induction machine current regulation and IFOC using integrated pilot current sensors.

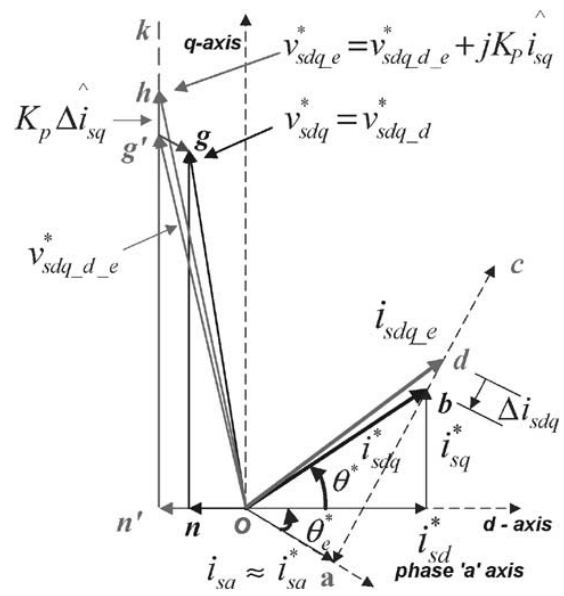

Fig. 5. Vector diagram showing commanded and actual current vectors plus commanded voltage vector in the presence of machine parameter errors.

Under these circumstances, the decoupling voltage inside the current regulator balances for the steady-state voltage of the machine and, as a result, the proportional controller develops zero voltage during steady-state conditions. This also implies that the steady-state stator current error is zero and the actual stator current vector $i_{s q d}$ exactly matches the commanded vector $i_{s d q}^{*}$ (vector $\left.o b\right)$.

Considering the case when the estimated stator resistance is larger that the actual stator resistance, both of the $d$ - and $q$-axes components of the decoupling voltage represented by vectors $o n^{\prime}$ and $n^{\prime} g^{\prime}$, respectively, become inaccurate and develop a resultant decoupling voltage represented by the vector $o g^{\prime}$, labeled $v_{s d q \_d \_}^{*}$ in Fig. 5. Since this voltage vector does not match the required steady-state voltage (vector $o g$ ), the proportional controller becomes effective to reduce the result- ing current error. However, assuming operation under constant $d$-axis current, the proportional controller voltage is limited to developing extra voltage along the $q$-axis, so the tip of the commanded voltage vector must locate at some point on the line $n^{\prime} k$ (the extension of line $n^{\prime} g^{\prime}$ ). In Fig. 5, this voltage command corresponds to $v_{s d q \_e}^{*}$ (vector $\left.o h\right)$.

This new voltage command causes the stator current vector labeled $i_{s d q \_}($vector $o d$ ) to flow. The proportional controller gain is assumed to be sufficiently high so that the steady-state current error in phase $a$ will be driven to nearly zero according to (3). As a result, the tip of the stator current vector od must lie somewhere on the line $a c$ (i.e., a line perpendicular to the phase $a$ axis passing through the tip of the commanded phase $a$ current value $i_{s a}^{*}$ ).

In addition to the two controller-imposed constraints described above, the commanded voltage vector and the actual stator current vector must satisfy the steady-state portion of machine equation (1) both in magnitude and angle. Combining these constraints, it can be shown that, for any erroneous decoupling voltage vector $v_{s d q_{d} d_{-}}^{*}$, a unique stator current vector exists (along line $a c$ ), which results in errors in the two unmeasurable phase currents. Simply adding an integral term to the proportional controller will not eliminate these errors, since an infinite proportional gain has already been assumed.

However, the back EMF portion of $q$-axis decoupling voltage lies entirely along the $q$-axis and the parameter errors associated with this voltage do not result in significant current errors, because the $q$-axis proportional controller can drive the voltage vector command to the desired value of decoupling voltage (vector $o g$ ). In contrast, parameter errors associated with either stator resistance or stator transient inductance will result in voltage errors in both $d$ - and $q$-axes decoupling voltage errors and, in this situation, the $q$-axis proportional controller will 

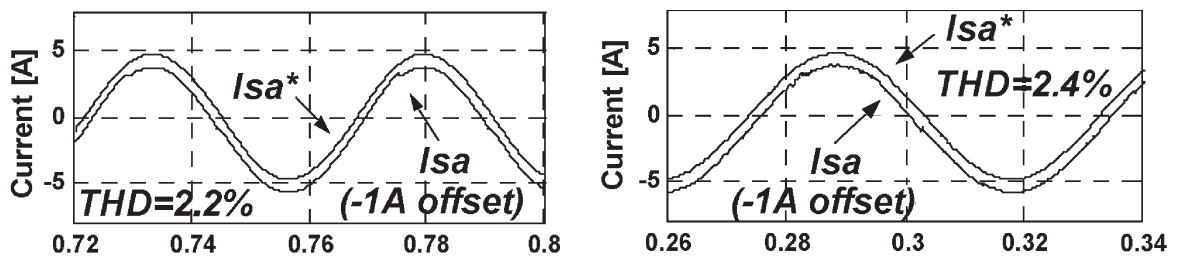

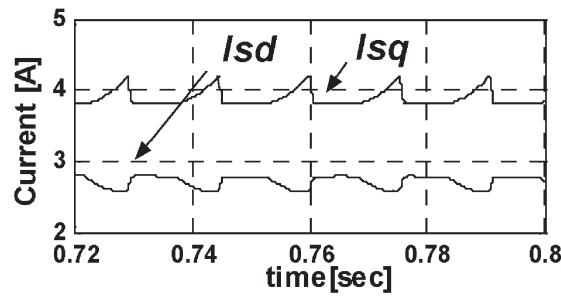

(a)

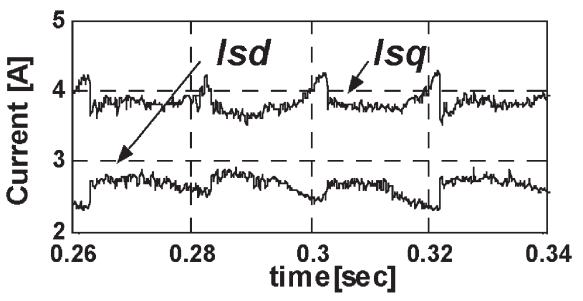

(b)

$$
\Delta\left(\hat{\sigma L_{s}}\right)=+25 \% \sigma L_{s}
$$

Fig. 6. Simulation and experimental results showing the effects of errors in stator transient inductance; $i_{s d}^{*}=2.8 \mathrm{~A}$ and $i_{s q}^{*}=3.8 \mathrm{~A}$. (a) Simulation. (b) Experimental.

only be able to compensate for the $q$-axis voltage error but will fail to compensate for the $d$-axis decoupling voltage error. This causes the proposed algorithm to be sensitive to the estimation of stator resistance and stator transient inductance, which results in errors in the unmeasurable phase currents during the 60 electrical degree intervals that have a single-phase current measurement.

Sensitivity of the algorithm to parameter estimation errors has been studied using both analytical and experimental techniques. Simulation results are presented in Fig. 6(a), showing the impact of estimation errors in the stator transient inductance. The top pair of traces compare the commanded phase current with the simulated phase current (with -1 A offset added for easier comparison) and the bottom traces represent the synchronous-frame $d q$ currents in the same format but used a different current scale than the top ones for better visibility of the ripple content. These simulation results indicate that the phase currents in both the stationary and synchronous frames develop harmonic distortion because of errors in the stator transient inductance, and that errors in the stator resistance produce similar effects. Although not shown here, this distortion also creates ripple in the instantaneous torque.

Experimental tests were carried out under the same operating conditions, and the corresponding waveforms are presented in Fig. 6(b). The characteristics of these waveforms correspond well to those in the simulated waveforms of Fig. 6(a). The simulation and the experimental results confirm that parameter errors in the stator leakage reactance and resistance both degrade the performance of the proposed synchronous-frame current regulator with the $60^{\circ}$ estimation intervals.

\section{InClusion of A GAted Integral Control Term}

The fact that all three phase currents are measurable during three of the $60^{\circ}$ intervals during each cycle makes it possible to use this extra feedback information to compensate for parameter errors during intervals when only one phase current is measurable. More specifically, a gated integral control term can be added to the current regulator to reduce the regulator sensitivity to inaccurate parameter estimates that cause errors in the steady-state decoupling voltages in (2).

The integrator is activated during each of the intervals when all of the three phase currents are measurable, responding as the integrator in a conventional synchronous-frame PI-type controller [7]. The computation of this integral term is carried out using the measured phase currents transformed into the synchronous frame

$$
v_{s d q \_i}^{*}=\frac{K_{I}}{s}\left(i_{s d q}^{*}-i_{s d q}\right)=\frac{K_{I}}{s} \Delta i_{s d q} .
$$

This integral controller term accumulates output amplitude due to the errors in the synchronous-frame currents during each of the intervals when all of the phase currents are measurable. The integrator is then frozen during each subsequent interval when only one phase current is measurable because of the absence of complete current feedback. That is, the integral voltage command is held at the last value computed during the preceding $60^{\circ}$ interval when all of the phase currents were measurable.

This integral voltage command term supplements the decoupling voltage calculated using (2) to command a more nearly correct value of steady-state motor voltage during the intervals when only a single-phase current is measurable. Thus, the modified version of the complete synchronous-frame voltage command including this integral term is

$$
v_{s d q}^{*}=K_{P} \Delta \hat{i_{s d q}}+\left(v_{s d q \_d}^{*}+v_{s d q \_i}^{*}\right) \text {. }
$$

With the inclusion of this integral term, the steady-state voltage demanded by the motor load is more accurately developed by the controller even in the presence of machine parameter errors. Thus, the integral term compensates the error in the decoupling voltage represented by the vector $g^{\prime} g$ in Fig. 5 so 


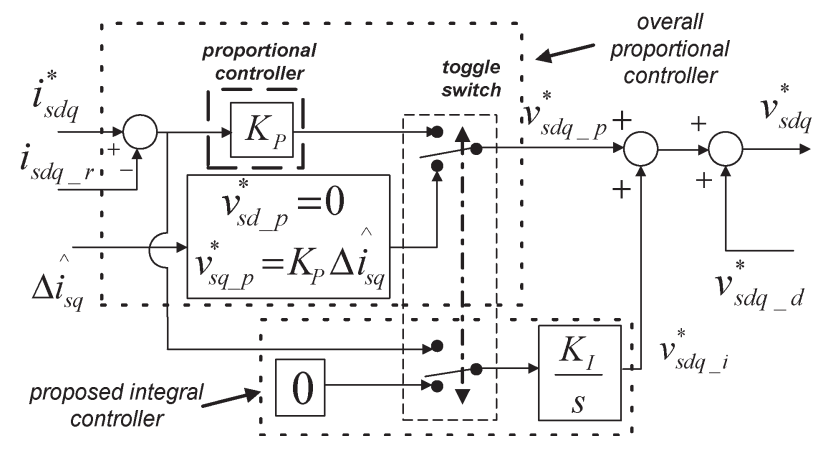

Fig. 7. Block diagram showing the inclusion of the proposed integral term in the synchronous-frame current controller.

that the stator current vector $i_{s d q}$ can more closely match the commanded vector $i_{s d q}^{*}$.

The proposed integral term described above has been incorporated into the controller structure in the experimental test equipment. Fig. 7 shows the modifications affecting a portion of the control block diagram in Fig. 4. This block diagram shows that the gated integral controller integrates the synchronousframe current error during the intervals with complete phase current feedback. In contrast, it integrates numerical value zero during the other three intervals representing hold action. Switching between these two inputs is controlled by the same "toggle switch" that selects the appropriate proportional controller voltage in Fig. 4.

Both simulation and experimental tests results have been used to evaluate the performance improvement achieved by the inclusion of the gated integrator term. Simulated results are presented in Fig. 8(a), considering a $25 \%$ error in the stator transient inductance, the same test conditions addressed in Fig. 6. The results in the first row compares the commanded phase- $a$ current with the actual machine current (with an added -1 A offset) in the same phase. The second row represents the synchronous-frame currents. The third row shows the machine torque estimated using the synchronous-frame currents in the second row with estimated machine parameters.

Experimental results in Fig. 8(b) are produced for the same test conditions, with the same set of displayed waveforms. In each of the cases, the stationary-frame measured current shows very little evidence of harmonic distortion. Comparison of the waveforms in Figs. 6 and 8 makes it clear that the integral term is effective under steady-state conditions for compensating the effects of parameter errors.

Dynamic test conditions have also been investigated using step changes in the amplitude of the stator current command. This test is conducted only with varying $q$-axis component of current, keeping the $d$-axis current constant. Fig. 9(a) shows the experimental test results for a parameter error of $+25 \%$ in the stator transient leakage inductance without the integral term in the controller. The first row compares the measured $q$-axis current (with -1 A offset added) with the stepped command current, while the second row compares the measured $d$-axis current (with -1 A offset added) with the commanded current. The third row compares the estimated torque calculated using estimated motor parameters and the synchronousframe currents in the first and second rows. The fourth row magnifies a portion of the synchronous-frame machine currents (without any offset) and provides a better visibility of the ripple components.

In contrast, test results in Fig. 9(b) are generated with the same test conditions except for incorporating the gated integrator into the controller structure. The test results follow the same format as in Fig. 9(a). Test results in Fig. 9(b) indicate that the ripple component in each of the waveforms has been reduced significantly. Comparison between these two sets of results confirms that the presence of the gated integral term improves the dynamic performance, delivering very good dynamic response even in the presence of significant machine parameter errors.

\section{Light-LoAd CURRENT RECONSTRUCtion}

As discussed in Section I, the reciprocal sine function in (3) causes a computational singularity [6], which affects the current controller performance during light-load conditions when the commanded synchronous-frame current components satisfy the inequality

$$
i_{s q}^{*}<\frac{i_{s d}^{*}}{\sqrt{3}}
$$

Fig. 10 shows the 60 electrical degree interval (with only phase $a$ current measurable) having such a light-load condition. This figure illustrates a time instant when the commanded stator current vector is located inside this interval with the $d$-axis oriented along $\theta_{e}^{*}=\pi$. Conversely, any load condition satisfying $i_{s q}^{*}>i_{s d}^{*} / \sqrt{3}$ places the stator current vector outside this sector into a region where all three phase currents are measurable, making the evaluation of (3) unnecessary. Similar conditions also occur during the other two $60^{\circ}$ intervals with single measurable currents.

Since the reciprocal sine function is infinite at $\theta_{e}^{*}=\pi$, a different current regulation algorithm is proposed for activation within a small angular sector around this singularity point. Inside this region, a simplified model of the current controller can be developed. It is assumed that the steady-state voltage decoupling is nearly ideal because of the combination of decoupling voltage using (2) plus the integral controller voltage described in the preceding section. Under these circumstances, the proportional controller is primarily responsible for determining the current vector trajectory, so the $q$-axis stator current responds to commands as a simple first-order lag as shown in the block diagram in Fig. 11. The $d$-axis current command is assumed to be constant.

Based on this simplified model, the synchronous-frame current components can be estimated as

$$
\begin{aligned}
& \hat{i}_{s q}=\frac{K_{P}}{K_{P}+s\left(\sigma \hat{L}_{s}\right)} i_{s q}^{*} \\
& \hat{i}_{s d}=i_{s d}^{*} .
\end{aligned}
$$

The angle of this estimated current vector $\hat{i}_{s d q}=\hat{i}_{s d}+j \hat{i}_{s q}$ with respect to the rotating $d$-axis $(\hat{\theta})$ is then used to reestimate 

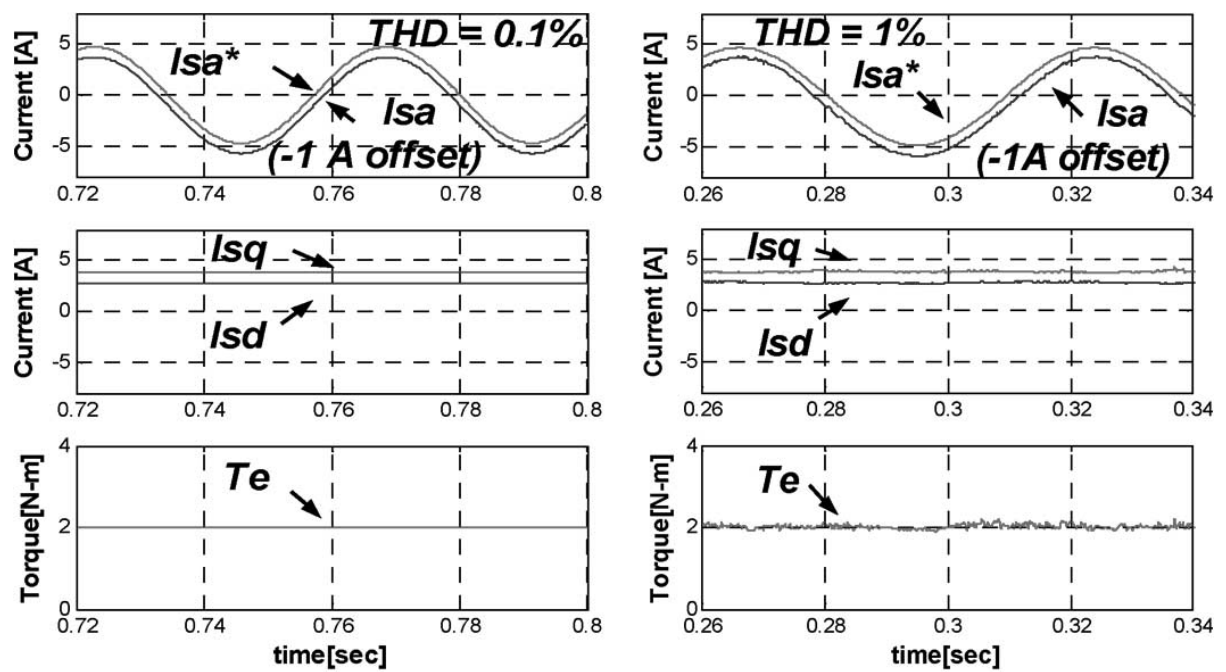

(a)

(b)

$$
\Delta\left(\hat{\sigma L_{s}}\right)=+25 \% \sigma L_{s}
$$

Fig. 8. Simulation and experimental results showing the effects of gated integrator with inductance parameter error; $i_{s d}^{*}=2.8$ A and $i_{s q}^{*}=3.8$ A. (a) Simulation. (b) Experimental.

the synchronous-frame currents using the only measurable phase current from phase $a$

$$
\hat{i}_{s d q \_r}=\frac{i_{s a \_m} e^{j \hat{\theta}}}{\cos \left(\theta_{e}^{*}+\hat{\theta}\right)}
$$

where $\hat{\theta}=\tan ^{-1}\left(\hat{i}_{s q} / \hat{i}_{s d}\right)$.

The estimated synchronous-frame currents from (11) are used to close the current feedback loop within a narrow angular span around $\theta_{e}^{*}=\pi$. The same approach can be applied inside the other two intervals with single measurable phase currents in the vicinity of their singularity point.

The proposed technique has been integrated into the original control structure so that it is activated only during a small angle span as described above. Experimental results are shown in Fig. 12 for the case when the new estimator is activated within an angular span of 20 electrical degrees around the point of singularity in each of the three $60^{\circ}$ intervals when only a singlephase current is measurable.

The first row in Fig. 12 shows the three phase currents, while the second row compares the measured phase $a$ current (with -1 A offset added) with the commanded current using the best available machine parameters. In contrast, test results in Fig. 12(b) show the same set of waveforms in the same format with $+30 \%$ error in the stator leakage inductance. These test results confirm that the proposed technique performs very well under light-load conditions.

\section{Compens ation of SEnsor Gain Mismatch}

Pilot current sensors offer some attractive performance characteristics including excellent bandwidth and temperature in- sensitivity characteristics [5]. However, as noted in Section I, these pilot sensors suffer from a vulnerability to significant variations in the current gain from device to device. Three pilot devices in the low-side inverter switches having differences in current gains will result in torque ripple that is clearly undesirable.

\section{A. Compensation Technique Derivation}

An effective technique has been developed to compensate differences in the relative current gains of the three pilot devices used for phase current sensing in the Fig. 1 configuration. It is assumed that the load is three-wire (no neutral). The approach is not useful for determining the absolute current sensor gains.

The technique consists of electronically configuring the three-phase load as an equivalent single-phase load by selecting proper modulating voltage commands without any necessity of physically reconnecting the stator phase terminals. The effectiveness of this approach is not degraded by imbalances in the phase load impedances.

For machine loads, it is assumed that the rotor is stationary so that any back-EMF voltage is absent, reducing the nature of the load to $R L$. The test procedure requires two different single-phase load configurations. The voltage modulator runs in open loop and delivers three-phase unbalanced voltages across the stator terminals at an arbitrary predetermined frequency so that the currents through the phase winding do not exceed the inverter device ratings.

The first load configuration is achieved by assigning identical voltage commands to phases $b$ and $c$ so that $v_{s b c}^{*}$ is zero. That is, the phase $b$ and $c$ terminals are effectively short circuited as shown in Fig. 13(a) so that $v_{s a b}^{*}=v_{s a c}^{*}$. 


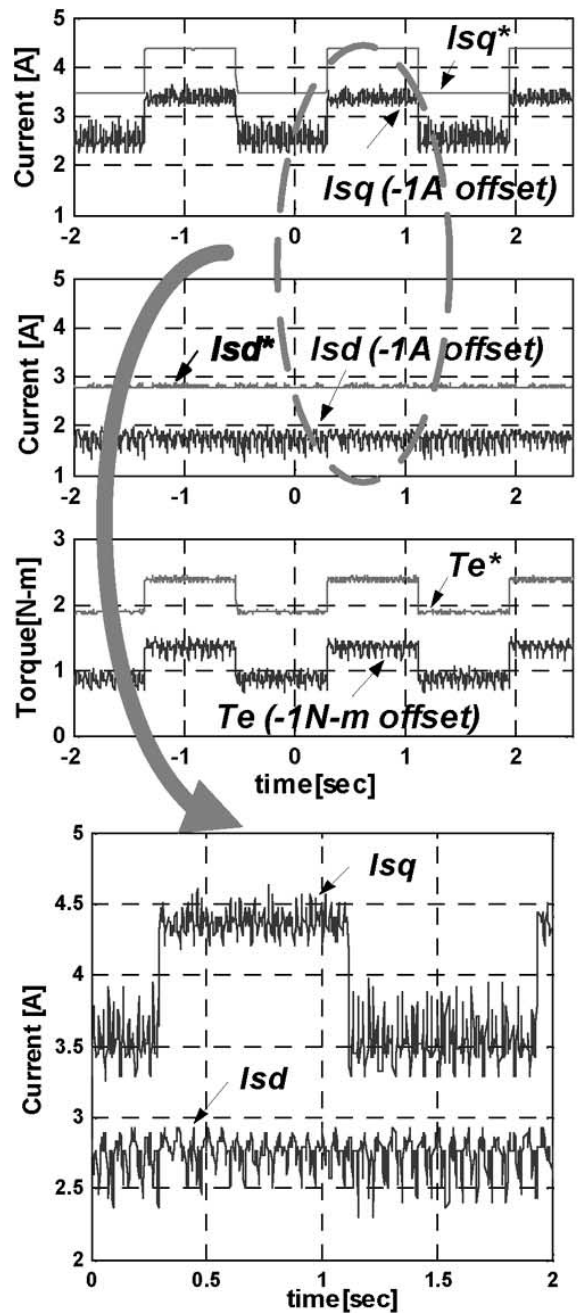

(a)
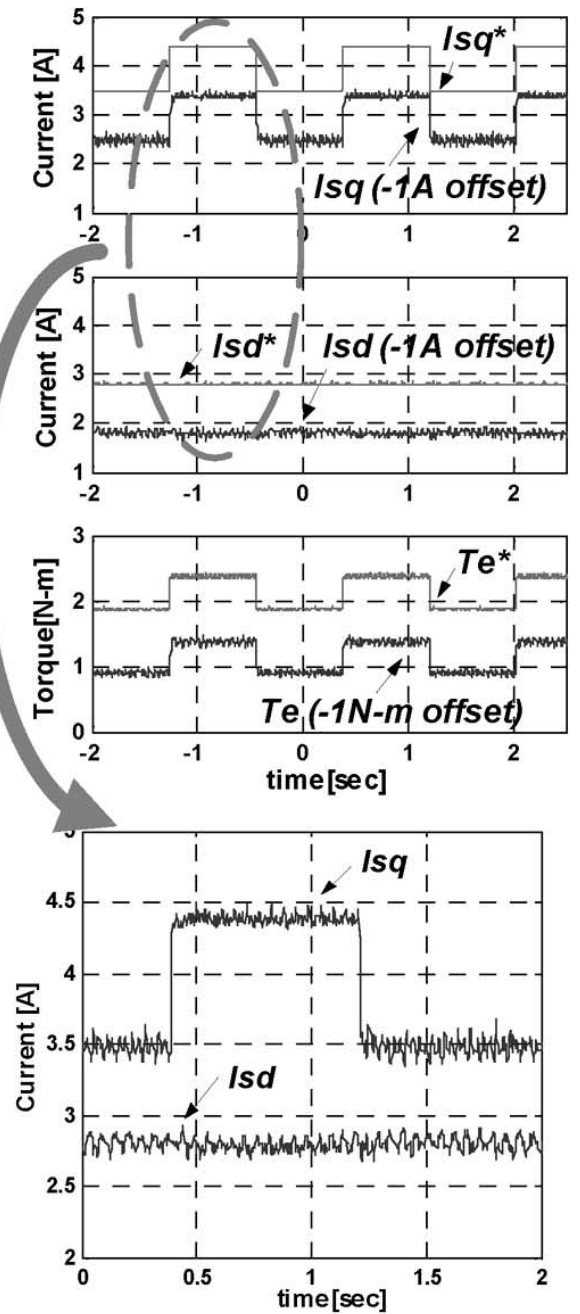

(b)

$\Delta\left(\hat{\sigma L_{s}}\right)=+25 \% \sigma L_{s}$

Fig. 9. Experimental results showing the dynamic performance of current controller with step changes in $i_{s q}^{*}$ between $3.4-4.4$ A; $i_{s d}^{*}=2.8$ A. (a) Without integral term. (b) With integral term.

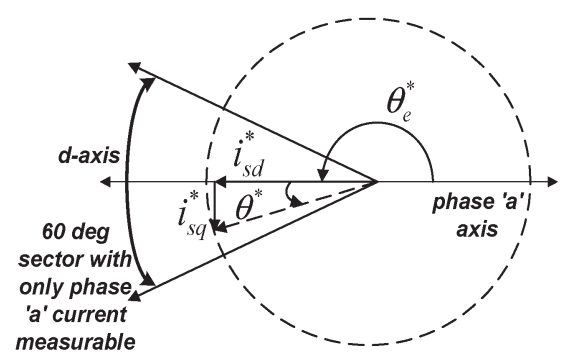

Fig. 10. Vector diagram showing the location of the commanded stator current vector at $\theta_{e}^{*}=\pi$ for light-load conditions.

The PWM voltage modulator can then be used to apply a desired single-phase alternating current (ac) voltage across the terminals of this load configuration. Since the instantaneous sum of $i_{s b}$ and $i_{s c}$ must be equal to the negative of the instantaneous phase $a$ current $i_{s a}$, this relationship can be written

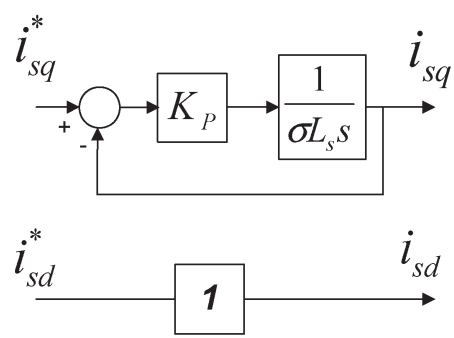

Fig. 11. Approximated simplified block diagram for the overall control and the physical system.

in phasor form in terms of the pilot sensor measurements and sensor gains

$$
\frac{I s a \_m \_1}{G_{a}}=-\frac{I_{s b \_m \_1}}{G_{b}}-\frac{I_{s c \_m \_1}}{G_{c}}
$$



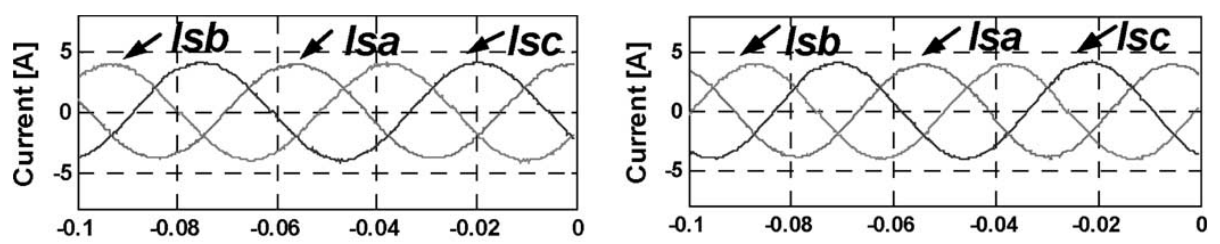

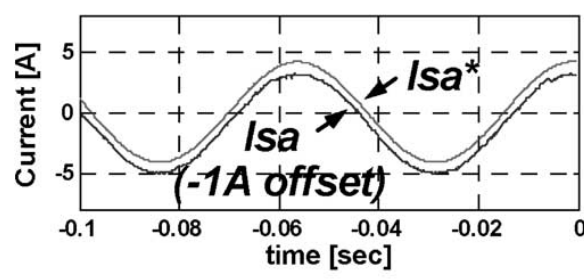

(a)

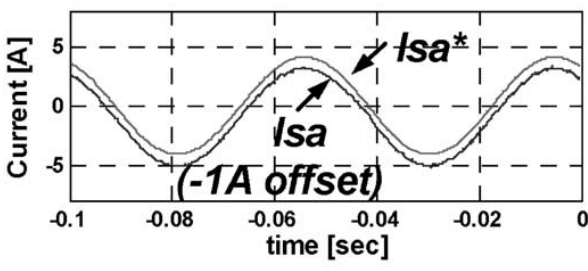

(b)

Fig. 12. Experimental results with the modified controller for light-load operation; $i_{s d}^{*}=3.7 \mathrm{~A}$ and $i_{s q}^{*}=1.8 \mathrm{~A} .(\mathrm{a}) \Delta\left(\sigma \hat{L}_{s}\right)=0 \% \sigma L_{s} .(\mathrm{b}) \Delta\left(\sigma \hat{L}_{s}\right)=$ $+30 \% \sigma L_{s}$.

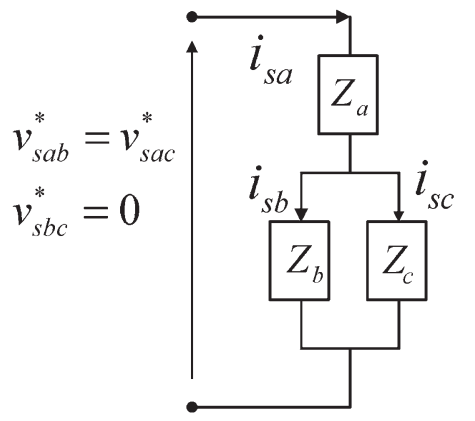

(a)

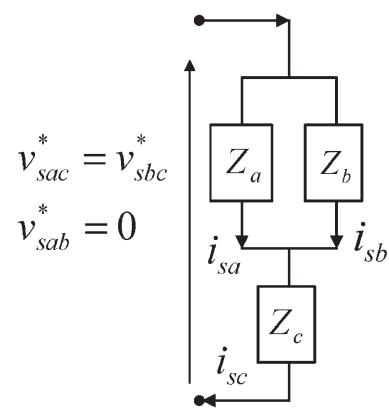

(b)
Fig. 13. Two single-phase configurations of a three-phase load. (a) Configuration 1. (b) Configuration 2.

where $I_{s a \_m \_1}, I_{s b \_m \_1}$, and $I_{s c \_m \_1}$ represent the measured phasors for the three phase currents in the Fig. 13(a) configuration. $G_{a}, G_{b}$, and $G_{c}$ are the gains of the pilot sensors including the signal processing circuits in the three phases, respectively.

Fig. 13(b) shows the second single-phase load configuration that uses the PWM modulator to effectively short circuit phases $a$ and $b$ so that $v_{s a c}^{*}=v_{s b c}^{*}$. As was done in the first configuration, a single-phase ac voltage is applied across the terminals of this second load configuration. Thus, the phasor relationship between the measured currents can be written as

$$
\frac{I_{s a \_m \_2}}{G_{a}}=-\frac{I_{s b \_m \_2}}{G_{b}}-\frac{I_{s c \_m \_2}}{G_{c}} .
$$

Since the objective is to find out the relative gains of the three pilot devices, the current gain of the phase $a$ pilot device can be selected as the baseline. The current gains of the pilot devices in phase $b$ and phase $c$ can then be determined as normalized quantities using the phase- $a$ current gain as the base quantity.

Each of the two phasor equations (12) and (13) can be turned into real algebraic equations by extracting the components of the phasors oriented along $I_{s a \_} m_{-} 1$ and $I_{s a \_m \_}$, respectively. The resulting two equations are combined in matrix form, with the four matrix elements expressed in terms of phasor magnitudes and cosines of the angles between the phasors

$$
\begin{aligned}
& {\left[\begin{array}{l}
\left|I_{s a \_m \_1}\right| \\
\left|I_{s a \_m \_2}\right|
\end{array}\right]} \\
& =\left[\begin{array}{ll}
-\left|I_{s b \_m \_1}\right| \cos \left(\theta_{b a \_1}\right) & -\left|I_{s c \_m \_1}\right| \cos \left(\theta_{c a \_1}\right) \\
-\left|I_{s b \_m \_2}\right| \cos \left(\theta_{b a \_2}\right) & -\left|I_{s c \_m \_2}\right| \cos \left(\theta_{c a \_2}\right)
\end{array}\right]\left[\begin{array}{c}
\frac{1}{G_{b}} \\
G_{a} \\
\frac{1}{G_{c}}
\end{array}\right] \text {. }
\end{aligned}
$$

After performing the two tests to determine the magnitudes of the phasors and their relative angles, the relative current gains of the pilot devices in phases $b$ and $c$ defined as $G_{b} / G_{a}$ and $G_{c} / G_{a}$, respectively, can be determined by solving (14).

\section{B. Compensation Technique Verification}

For purposes of experimental verification, an additional software scaling factor was introduced for each of the three pilot sensor feedback signals so that the total current sensor gain $G$ can be expressed as

$$
G_{x}=G_{x}^{\prime} \cdot H_{x}
$$

where $G^{\prime}$ is the gain of the pilot sensor and its signalconditioning electronics, $H$ is the software scaling factor, and $x=a, b, c$.

The experimental test equipment was used to record the phase currents measured by the three pilot sensors in the two configurations shown in Fig. 13. Since each of the pilot devices can only measure the negative half-cycle of its respective phase current, the positive half-cycle was synthesized by inverting the measured negative half-cycle.

Two separate experiments were conducted. In the first experiments, the $H$ scaling factors for all three phases were all set to unity. Current waveforms for the two test configurations that include the impact of both the $G^{\prime}$ and $H$ factors in (15) are provided in Fig. 14(a). The results of solving (14) using these test results are shown at the bottom of Fig. 14(a), showing that the calculated pilot current sensor gains for phases $b$ and $c$ are each $1 \%$ higher than the phase- $a$ sensor gain. 

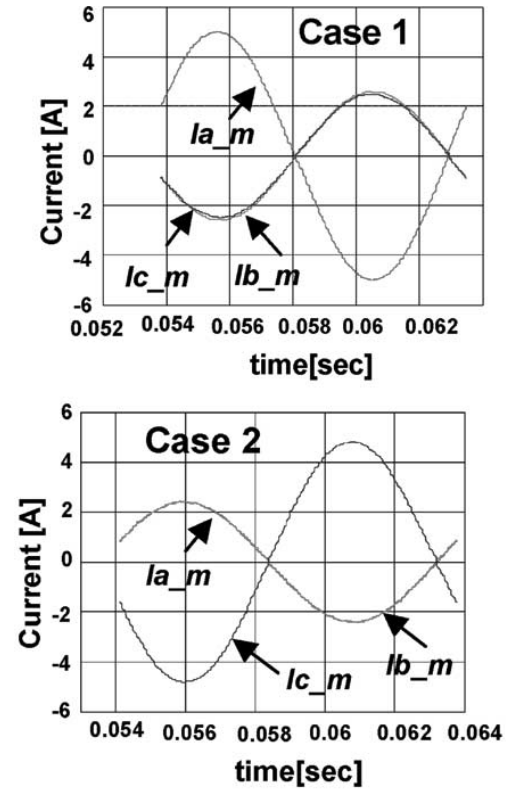

$H_{b} / H_{a}=1.00, H_{c} / H_{a}=1.00$

$G_{b} / G_{a}=1.01, G_{c} / G_{a}=1.01$

$G_{b}^{\prime} / G_{a}^{\prime}=1.01, G_{c}^{\prime} / G_{a}^{\prime}=1.01$

(a)

Fig. 14. Experimental test results for the sensor gain compensation technique.

During the second test, the $H$ scaling factor for phase $b$ was lowered by $5 \%$, while the corresponding factor for phase $c$ was raised by $5 \%$. The current waveforms in Fig. 14(b) were collected for the two test configurations, and the effects of the current gain differences can be observed. The results of solving (14) for the current gains are summarized at the bottom of the figure, and they are very encouraging. The calculated values for the current sensor gains $\left(G^{\prime}\right)$ match the values calculated in the previous test despite the artificial detuning introduced by the scaling factors in phases $b$ and $c$.

These test results provide experimental verification that the proposed technique can compensate for relative differences in the pilot sensor current gains.

\section{CONCLUSION}

This paper has presented a new approach for delivering attractive current-regulated performance characteristics for threephase induction motors using only three integrated pilot current sensors embedded in the low-side inverter switches. The original version of this current regulator [6] has been modified by adding a gated integral controller so that the regulator behaves very well under both steady-state and dynamic test conditions despite the presence of significant errors in the estimated motor parameter values.

A known limitation of the original current regulator associated with light-load operating conditions has also been overcome. More specifically, a first-order estimator for the phase currents has been introduced, which is only activated over narrow angular spans in order to avoid a numerical singularity caused by a reciprocal sine term.
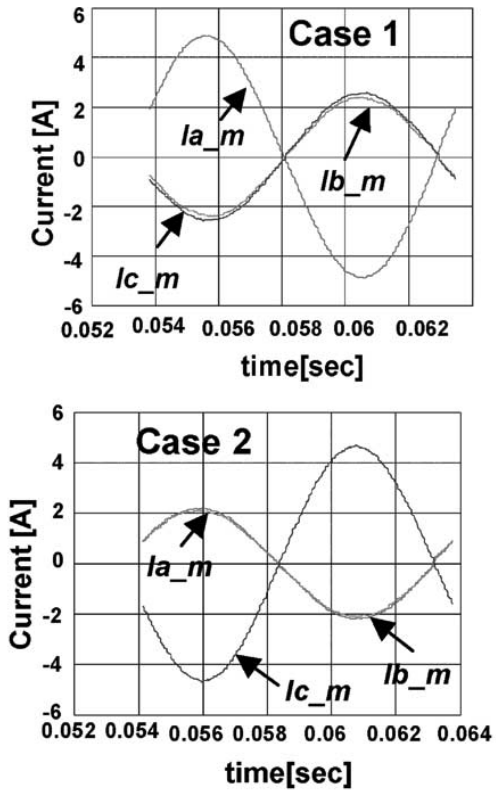

$H_{b} / H_{a}=0.95, H_{c} / H_{a}=1.05$

$G_{b} / G_{a}=0.96, G_{c} / G_{a}=1.06$

$G_{b}^{\prime} / G_{a}^{\prime}=1.01, G_{c}^{\prime} / G_{a}^{\prime}=1.01$

(b)

This paper also proposes a simple but effective technique for compensating the relative differences in the gains of the three pilot sensors in the low-side inverter switches. The technique is implemented using the PWM voltage modulator and is insensitive to imbalance in the three load phases.

Separately, work is continuing on improving the performance characteristics of the pilot current sensor devices. Assuming success in those efforts, the promising results presented here raise confidence that pilot sensors can be applied in future induction machine drives to achieve effective closed-loop current regulation for field-oriented control.

\section{REFERENCES}

[1] T. M. Jahns and E. J. Wildi, "Integrated current sensor configurations for AC motor drives," U.S. Patent 4777 579, Oct. 11, 1988.

[2] T. M. Jahns, R. C. Becerra, and M. Ehsani, "Integrated current regulation for a brushless ECM drive," IEEE Trans. Power Electron., vol. 6, no. 1, pp. 118-126, Jan. 1991.

[3] S. R. MacMinn, W. J. Rzesos, P. M. Szczesny, and T. M. Jahns, "Application of sensor integration techniques to switched reluctance motor drives," IEEE Trans. Ind. Appl., vol. 28, no. 6, pp. 1339-1344, Nov./Dec. 1992.

[4] Z. Shen, "Modeling, design, fabrication, and characterization of the insulated gate bipolar transistor (IGBT) with integrated current sensors," Ph.D. dissertation, Rensselaer Polytechnic Inst. (RPI), Troy, NY, 1994.

[5] S. Chakrabarti, T. M. Jahns, and R. D. Lorenz, "A current reconstruction algorithm for three-phase inverters using integrated current sensor as low side switches," in Conf. Rec. IEEE-IAS Annu. Meeting, Salt Lake City, UT, Oct. 2003, pp. 925-932.

[6] _ - "A current control technique for induction machine drives using integrated pilot current sensors in the low-side switches," in Proc. IEEE PESC'04, Aachen, Germany, Jun. 2004, pp. 4453-4459.

[7] D. W. Novotny and T. A. Lipo, Vector Control and Dynamics of AC Drives. New York: Oxford Univ. Press, 1996.

[8] T. R. Rowan and R. L. Kerkman, "A new synchronous current regulator and an analysis of current-regulated PWM inverters," IEEE Trans. Ind. Appl., vol. 22, no. 4, pp. 678-690, Jul./Aug. 1986. 


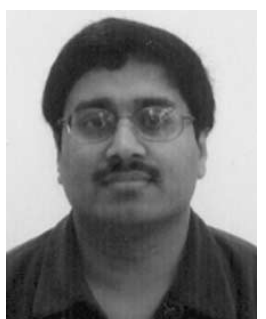

Sibaprasad Chakrabarti (S'01-M'04) received the B.E. degree in electrical engineering from the Bengal Engineering College, Howrah, Calcutta University, India, in 1992, the M.E. degree in electrical engineering from the Indian Institute of Science, Bangalore, India, in 1994, and the Ph.D. degree in electrical and computer engineering from the University of Wisconsin, Madison, in 2004.

From 1994 to 2000, he was with R. B. Data Comms (p) Ltd., Calcutta, India, and Asea Brown Boveri Ltd, Baroda, India. During this period, he was involved in various research-and-development-related activities in the area of power electronics and motor drives. After completion of the Ph.D. degree, he joined GE Transportation Systems, Erie, PA, where he focuses on power converter development activities for induction motor drives application.

Dr. Chakrabarti was one of the recipients of the First-Prize Paper Award of the IEEE Industry Applications Society (IAS) Industrial Drives Committee at the IAS 2003 Annual Meeting in Seattle, WA.

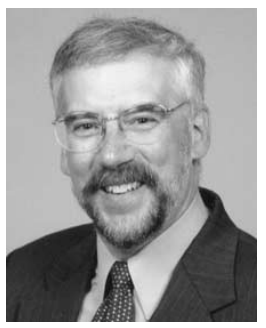

Thomas M. Jahns (S'73-M'79-SM'91-F'93) received the S.B. and S.M. degrees in 1974 and the $\mathrm{Ph} . \mathrm{D}$. degree in 1978 from the Massachusetts Institute of Technology, Cambridge, all in electrical engineering.

He joined the faculty of the University of Wisconsin, Madison (UW-Madison), in 1998 as a Grainger Professor of Power Electronics and Electric Machines in the Department of Electrical and Computer Engineering. He is an Associate Director of the Wisconsin Electric Machines and Power Electronics Consortium (WEMPEC). Prior to coming to UW-Madison, he was with GE Corporate Research and Development (now GE Global Research Center), Schenectady, NY, for 15 years, where he pursued new power electronics and motor drive technology in a variety of research and management positions. His current research interests include permanent-magnet synchronous machines and advanced features for future generations of integrated power electronics modules (IPEMs). During 1996-1998, he conducted a research sabbatical at the Massachusetts Institute of Technology, where he directed research activities in the area of advanced automotive electrical systems and accessories as Co-Director of an industry-sponsored automotive consortium.

Dr. Jahns is the recipient of the 2005 IEEE Nikola Tesla Award. He was awarded the William E. Newell Award by the IEEE Power Electronics Society (PELS) in 1999. He was recognized as a Distinguished Lecturer by the IEEE Industry Applications Society (IAS) during 1994-1995 and by the IEEE PELS during 1998-1999. He has served as President of PELS (1995-1996) and as Division II Director on the IEEE Board of Directors (2001-2002).

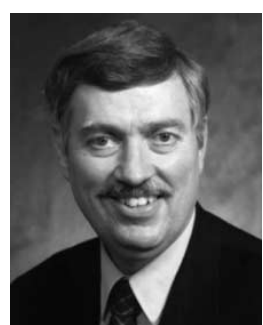

Robert D. Lorenz (S'83-M'84-SM'91-F'98) received the B.S., M.S., and Ph.D. degrees from the University of Wisconsin, Madison, and the M.B.A. degree from the University of Rochester, Rochester, NY.

Since 1984 , he has been a member of the faculty of the University of Wisconsin, Madison, where he is the Mead Witter Foundation Consolidated Papers Professor of Controls Engineering in both the Department of Mechanical Engineering and the Department of Electrical and Computer Engineering. $\mathrm{He}$ is Co-Director of the Wisconsin Electric Machines and Power Electronics Consortium, which will celebrate its 25th anniversary in 2006. It is the largest industrial research consortium on motor drives and power electronics in the world. $\mathrm{He}$ is also the thrust leader for control and sensor integration in the Center for Power Electronic Systems, an NSF Engineering Research Center which is a joint ERC with Virginia Polytechnic Institute, Rensselaer Polytechnic Institute, University of Puerto Rico-Mayaguez, and North Carolina A\&T. From 1972 to 1982 , he was a member of the research staff at the Gleason Works, Rochester, NY, working principally on high-performance drives and synchronized motion control. He was a Visiting Research Professor in the Electrical Drives Group of the Catholic University of Leuven, Leuven, Belgium, in the summer of 1989 and in the Power Electronics and Electrical Drives Institute of the Technical University of Aachen, Aachen, Germany, in the summers of 1987, 1991, 1995, 1997, and 1999, respectively, and was the SEW Eurodrive Guest Professor from September 1, 2000 until July 7, 2001. In 1969-1970, he did his Master's thesis research in adaptive control of machine tools at the Technical University of Aachen. His current research interests include sensorless electromagnetic motor/actuator technologies, real-time signal processing and estimation techniques, precision multiaxis motion control, and alternating current (ac)/direct current (dc) drive and high-precision machine control technologies. He has authored more than 170 published technical papers and is the holder of 23 patents with three more pending.

Dr. Lorenz is the IEEE Division II Director for 2005/2006, was the IEEE Industry Applications Society (IAS) President for 2001, a Distinguished Lecturer of the IAS for 2000/2001, past Chair of the IAS Awards Department, past Chairman of the IAS Industrial Drives Committee, and is a member of the IAS Industrial Drives, Electrical Machines, Industrial Power Converter, and Industrial Automation and Control Committees. He is the immediate past Chair of the Periodical Committee and current Chair of Periodicals Review Committee for the IEEE Technical Activities Board. He is a Member of the IEEE Sensor Council AdCom. He is a Registered Professional Engineer in the States of New York and Wisconsin. He is also a Member of the American Society of Mechanical Engineers, Instrument Society of America, and The International Society for Optical Engineers. He has won 18 prize paper awards. He was awarded the 2003 IAS Outstanding Achievement Award, which honors his outstanding contributions and technological developments in the application of electricity to industry. 